\title{
Evaluation of Macular Function Tests with Diabetic Retinopathy
}

\author{
Abhinav Ashok Agrawal ${ }^{1}$, Niharika Krishna Shetty ${ }^{2}$ \\ 1,2 Department of Ophthalmology, Sri Siddhartha Medical College Hospital and Research Center, Sri \\ Siddhartha Academy of Higher Education University, Tumakuru, Karnataka, India.
}

\section{ABSTRACT}

\section{BACKGROUND}

Diabetic retinopathy (DR) is reported to be the leading cause of vision loss in adults aged between 20-74 years. Early detection and prompt evaluation is essential to prevent the blindness related to diabetes. Simple and quick out-patient department (OPD) tests are essential for early detection of maculopathy in diabetes, which will enhance the treatment and rehabilitation. The purpose of this study was to evaluate the correlation of photo stress test and Amsler's grid test with diabetic retinopathy and maculopathy. We also wanted to study the variation in photo stress test and the patterns of visual disturbances using Amsler grid in different stages of diabetic retinopathy.

\section{METHODS}

All patients with type 2 diabetes were included for a study duration of one year. A cross sectional study design was planned. Anterior and posterior segment evaluation was done. Photo stress test was performed with a torch light and the recovery time was recorded. Amsler grid was performed on each patient at $33 \mathrm{~cm}$ distance. The results were recorded in terms of micropsia, macropsia, metamorphopsia, and any other ill-defined scotomas. The posterior segment, in terms of vitreous and retina was evaluated with $20 \mathrm{D}$ lens on an indirect ophthalmoscopy and the macular details were evaluated on a $90 \mathrm{D}$ lens with slit lamp biomicroscopy. Early Treatment Diabetic Retinopathy (ETDR) classification was used for classifying the retinopathy and the maculopathy stage in patients.

\section{RESULTS}

There was a correlation between paroxysmal supraventricular tachycardia (PST) and the stage of diabetic retinopathy; between PST and diabetic maculopathy; with increasing severity of diabetic retinopathy and maculopathy associated with higher or prolonged PST values. No correlation was found between Amsler's grid and DR staging.

\section{CONCLUSIONS}

PST can be used to assess severity of diabetic retinopathy in a pre-clinical and early clinical stage in places where access to the equipment for posterior segment evaluation is unavailable. Amsler's grid evaluation did not have a role in evaluation of macula in cases of diabetic retinopathy.

\section{KEY WORDS}

Macula, Amsler, Photostress Test, Metamorphopsia
Corresponding Author: Dr. Niharika Krishna Shetty, C-1403, Wing 1, Vaishnavi ARS Gardenia, Subrotho Mukherjee Road, Jallahalli (West),

Dasarahalli, Bangalore - 560057,

Karnataka, India.

E-mail: niharika.shetty30@gmail.com

DOI: $10.14260 / j e m d s / 2021 / 307$

How to Cite This Article:

Agrawal AA, Shetty NK. Evaluation of macular function tests with diabetic retinopathy. J Evolution Med Dent Sci 2021;10(20):1463-1468, 10.14260/jemds/2021/307

Submission 04-11-2020,

Peer Review 15-03-2021,

Acceptance 22-03-2021,

Published 17-05-2021.

Copyright (C) 2021 Abhinav Ashok Agrawal et al. This is an open access article distributed under Creative Commons Attribution License [Attribution 4.0 International (CC BY 4.0)] 


\section{BACKGROUND}

Diabetic retinopathy is reported to be the leading cause of vision loss all over the world. ${ }^{1}$ As well, it is said to be the leading cause of vision loss in adults aged $20-74$ years. ${ }^{2}$ In the past decade, diabetic retinopathy was noted as the fifth most common cause of preventable blindness and fifth most common cause of moderate to severe visual impairment. ${ }^{3}$ The causes of vision loss in diabetes were found to be severe in non proliferative diabetic retinopathy, proliferative diabetic retinopathy and diabetic macular oedema. ${ }^{4}$ Among these causes, proliferative diabetic retinopathy caused vision loss more frequently in type 1 diabetes whereas diabetic macular oedema was the frequent cause of vision loss in type 2 diabetes mellitus. ${ }^{5}$ Diabetic maculopathy is also reported to be invariably present in cases of proliferative diabetic retinopathy. 6

Diabetic macular oedema was defined clinically by hard exudates due to microaneurysms and blot hemorrhages within one disc diameter of the foveal center. Clinically significant macular edema (CSME) is defined by the presence of edema within $500 \mu \mathrm{m}$ of the foveal center. Hence, CSME was a more severe form of diabetic retinopathy. ${ }^{1}$ Many tools have been used to detect the incidence and prevalence of diabetic maculopathy. These methods include clinical methods to stereoscopic and non-stereoscopic fundus photographs to ocular coherence tomography (OCT). OCT-detected diabetic macular oedema (DME) was found to have a greater degree of disagreement with the clinical definition of CSME, and not all patients who had macular thickening detected on OCT progressed to have clinical DME. ${ }^{1}$

In contrast to diabetic retinopathy, the epidemiology of diabetic macular oedema is less studied. Existing studies are split between the use of two diagnostic criteria, one for DME and the other for CSME.The diagnosis of DME using this same modality is challenging as macular thickening is difficult to assess in non-stereoscopic photographs. There is no consensus on OCT based severity classification for DME. ${ }^{1}$

Macular edema is often associated with diabetic retinopathy severity. Diagnosis of DME in subjects is either following the manifestations of macular edema in a previously type 2 diabetes mellitus diagnosed individually, or some may even have decreased vision from macular edema as the presenting sign. However, a very small proportion of patients present like this as for most of the symptoms are masked. ${ }^{7}$

The pathopysiology of diabetic macular oedema is explained by the retinal circulation. Normal retinal circulation is unique: retinal capillaries are non-fenestrated and capillary endothelial cells have tight junctions. Normal capillaries do not leak fluid or blood. There is no lymphatic system in the retina so in the presence of retinal pathology, leaking fluid can accumulate and cause edema or swelling of the retinal layers. This also leads to macular oedema. ${ }^{7}$ Clinical manifestations of diabetic macular oedema are also varied.

Most of the patients whose central macula is spared are asymptomatic. Extremely observant patients may notice a paracentral scotoma corresponding to focal hard exudates and oedema in such cases. The central involvement of the macula of recent origin may also remain asymptomatic. If the oedema in the central macula persists, these patients may later complain of mild visual symptoms like gradual loss of vision, poor colour vision or poor dark adaptation. ${ }^{7}$
Clinically, the macula is evaluated on 90 diopters lens with slit lamp biomicroscopy and the macular oedema is classified as focal oedema due to cluster of microaneurysms, diffuse macular oedema due to loss of normal foveal contour and cystoids macular oedema due to appearance of cystic spaces on the macular area. ${ }^{7}$ Clinically significant macular edema is defined as one or more of the following: retinal thickening at or within $500 \mu \mathrm{m}$ of the center of the macula; hard exudates at or within $500 \mu \mathrm{m}$ of the center of the macula, if associated with adjacent retinal thickening; or a zone or zones of retinal thickening one disc area in size, at least part of which is within one disc diameter of the center of the macula. ${ }^{8}$

Methods of investigating the macular area include clinical evaluation, fluorecein angiography and ocular coherence tomography. The functional evaluation of macula is done with the various macular function tests such as Maddox rod test, photostress recovery test, blue-light entoptoscopy, Purkinje entoptic phenomenon and visual-evoked response and electroretinography (VER - ERG).

Photostress test was introduced by Bailliart (1954) who dazzled the macula with the light of an ordinary ophthalmoscope and measured recovery time of central vision. The basis of the test is to utilize an induced fatigue of the macula analogous to the induced fatigue utilized for the examination of the heart. The photostress test is a simple, easy and quick clinical technique that can differentiate between retinal (macular) and post-retinal (e.g.optic nerve) disease. ${ }^{9}$ The Amsler's grid is a type of macular function test used to detect the central 10 degrees of visual field, mainly in the area around fixation. It is a high contrast supra-threshold test.10,11 After correcting for near refractive error, each participant was asked to view each Amsler's grid $(10 \& 10 \mathrm{~cm})$ at a distance of $33 \mathrm{~cm}$, ensuring that each box on the Amsler's grid corresponds to 1 degree of visual field (VF). A similar high quality paper copy of the Amsler's grid was used for each patient. The Amsler's grid can be called as a type of visual field testing. ${ }^{12}$ Currently, clinical assessment of retinal function loss in diabetic maculopathy relies on visual acuity. Unfortunately, by the time visual acuity is reduced, the signs of retinal damage are already present.13,14,15 Many studies have shown that there are other psychophysical16,17,18,19,20 and electrophysiological tests that are based on contrast sensitivity to detect early vision loss in diabetics. ${ }^{21,22}$ However, these tests are time consuming and expensive.

In this study, we have tried to evaluate the macular function in diabetes using Amsler's grid and photo stress test, which are simple and fast OPD tests. These tests might help us to detect any subclinical functional loss if exists irrespective of anatomical changes found in maculopathy.

\section{METHODS}

All the diabetic patients visiting Department of Ophthalmology at SSMC,Tumkur, from May 2019 to May 2020 were included in the study. The ethical clearance was obtained from the institutional ethical committee. Letter reference number SSMC / IEC - 2 / April - 2019, dated 20 / 4 / 2019. The study design was a cross sectional study design. Detailed ocular evaluation was done including anterior segment and the posterior segment. Visual acuity was recorded on Snellen's visual acuity 
chart for distance. Near acuity was evaluated on times roman near vision chart and recorded. Photo stress test was performed with an ophthalmoscope light according to the standard protocol and the recovery time was recorded in seconds. Amsler's grid was provided and performed on each patient in an illuminated room at $33 \mathrm{~cm}$ distance. The results were recorded in terms of micropsia, macropsia, metamorphopsia and any other ill-defined scotomas. The black grid on white Amsler's was used in our study. The posterior segment in terms of vitreous and retina was evaluated with 20 diopter lens on an indirect ophthalmoscopy and the macular details evaluated on a $90 \mathrm{D}$ lens with slit.

Lamp biomicroscopy ETDRs classification was used for classifying the retinopathy and the maculopathy stage in the patients.

\section{Inclusion Criteria}

All patients diagnosed with type 2 diabetes mellitus.

\section{Exclusion Criteria}

1. Patients with cataract and glaucoma.

2. Patients with type 1 diabetes mellitus, hypertensive retinopathy.

3. Patients with age related macular degeneration.

4. Patients with any other maculopathy.

\section{Statistical Analysis}

The data was collected and statistical analysis was done. The data collected was subjected to chi-square test statistical analysis and the conclusions were drawn.

\section{RESULTS}

In our study group, we had 42 males, (59.155 \%) and 29 (40.845 \%) females. Since it was a hospital based study, we had more male patients presenting to us for medical help. In our study, the maximum cases were between 55 to 65 years of age group. There were almost 32 cases in that age group amounting to around $45.07 \%$ of cases. In our study, we had maximum patients presenting with the history of 10 to 15 years of diabetes. There were a total of 26 (36.6\%) patients in our study group who had such long standing history of diabetes.

In our study, we found that diabetic retinopathy was seen in $36(50.70 \%)$ cases. There was no evidence of diabetic retinopathy in 35 (49.29\%) cases. Amongst the retinopathy cases, the moderate type of diabetic retinopathy cases were highest amounting to $21(29.577 \%)$ cases. In our study we had $3(4.22 \%)$ patients with macular oedema qualifying for clinically significant macular oedema, and 1 (1.40\%) patient had cystoids macular oedema. Whereas 67 (94.366 \%) cases had no evidence of macular oedema on clinical evaluation with a 90 D lens. We had not performed OCT test on our study population to pick up sub clinical macular involvement.

In our study we had almost 37(52.11\%) patients who had a PST ranging between 11 seconds to 20 seconds, though this is considered to be a normal PST. In our study population, the abnormal PST (more than 50 seconds) was found only in 6 patients $(8.45 \%)$.

\begin{tabular}{|cccccc|}
\hline $\begin{array}{c}\text { Age in } \\
\text { Years }\end{array}$ & $\begin{array}{c}\text { Count - Age } \\
\text { in Years }\end{array}$ & $\begin{array}{c}\text { Percentile } \\
\text { Distribution }\end{array}$ & Gender & Frequency & $\begin{array}{c}\text { Percentile } \\
\text { Distribution }\end{array}$ \\
$31-40$ & 2 & 2.8 & Male & 42 & 59.14 \\
$41-50$ & 20 & 28.1 & Female & 29 & 40.84 \\
$51-60$ & 28 & 39.43 & & & \\
$61-70$ & 16 & 22.53 & & & \\
$71-80$ & 4 & 5.6 & & \\
$81-90$ & 1 & 1.4 & & \\
\hline \multicolumn{5}{c}{ Table 1. Age and Gender Distribution of } \\
Diabetes in Our Study Population
\end{tabular}

\begin{tabular}{|cccc|}
\hline ETDRS & Frequency & Diabetic Maculopathy & Frequency \\
No DR & 35 & No macular oedema & 67 \\
Mild DR & 10 & Clinically significant macular & 3 \\
oedema & 1 \\
Moderate DR & 21 & Cystoid macular oedema & \\
PDR & 4 & & \\
\hline \multicolumn{4}{|c|}{$\begin{array}{c}\text { Table 2. Distribution of ETDRS Prototype of } \\
\text { Diabetic Retinopathy and Maculopathy }\end{array}$} \\
\hline
\end{tabular}

\begin{tabular}{|c|c|c|c|c|c|c|c|c|}
\hline \multicolumn{9}{|c|}{ Contingency Tables } \\
\hline \multirow{2}{*}{$\begin{array}{c}\text { Diabetic } \\
\text { History in } \\
\text { Years }\end{array}$} & \multicolumn{8}{|c|}{ PST in Seconds } \\
\hline & \multicolumn{8}{|c|}{$10-2021-3031-4041-5051-6071-8081$ - 90 Total } \\
\hline$<5$ & 26 & 6 & 1 & 0 & 2 & 0 & 0 & 35 \\
\hline $6-10$ & 11 & 7 & 6 & 0 & 0 & 0 & 1 & 25 \\
\hline $11-15$ & 4 & 0 & 2 & 1 & 1 & 1 & 1 & 10 \\
\hline $16-20$ & 0 & 0 & 0 & 1 & 0 & 0 & 0 & 1 \\
\hline Total & 41 & 13 & 9 & 2 & 3 & 1 & 2 & 71 \\
\hline \multicolumn{9}{|c|}{$\begin{array}{l}\text { Table 3. Comparison of Diabetic History Duration with } \\
\text { Photostress Test. Statistical Analysis with Chi -Square Test }\end{array}$} \\
\hline$P-$ Value $<0$ & & & & & & & & \\
\hline
\end{tabular}

The $\mathrm{P}$ value was significant for all in a higher range of seconds in patients with longer duration of diabetes. The chisquare test $P$ value was less than 0.001 proving that the longer the duration of diabetes, higher was the value of PST in seconds, though the values were still within the normal range. In our study population, the abnormal PST (more than 50 seconds) was found only in 6 patients (8.45\%).

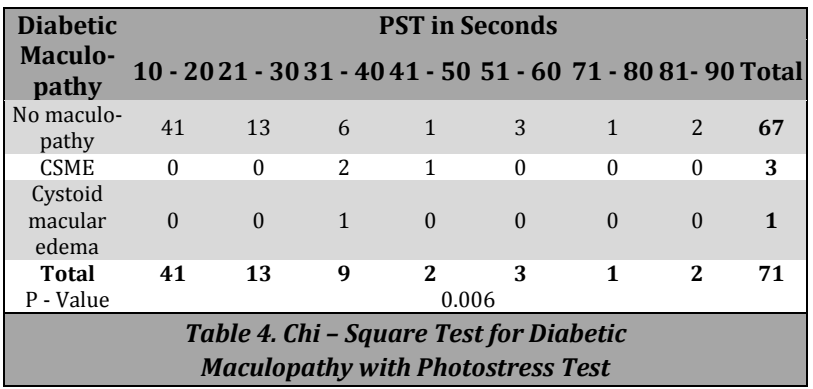

We found that the PST did not correlate significantly with the maculopathy. (P-Value was 0.006). But the cases of maculopathy did show a prolonged PST.

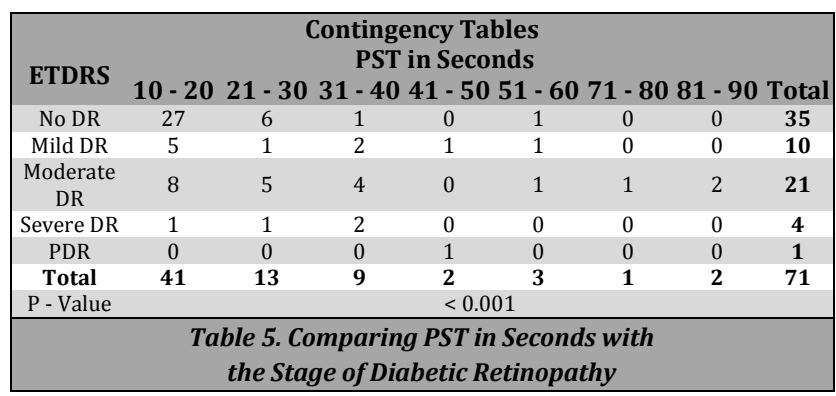


We found that patients with moderate diabetic retinopathy had the maximum cases of abnormal PST, approximately 4 (19.04 \%) patients having abnormal PST amongst 21 cases.

\begin{tabular}{|c|c|c|c|}
\hline ETDRS & Normal & $\begin{array}{r}\text { Amsler's Grid } \\
\text { Metamorphopsia }\end{array}$ & Total \\
\hline No DR & 35 & 0 & 35 \\
\hline Mild DR & 10 & 0 & 10 \\
\hline Moderate DR & 19 & 2 & 21 \\
\hline Severe DR & 4 & 0 & 4 \\
\hline PDR & 1 & 0 & 1 \\
\hline Total & 69 & 2 & 71 \\
\hline$P$ - Value & & 0.298 & \\
\hline \multicolumn{4}{|c|}{ Comparison of Diabetic Retinopathy with Amsler's Grid } \\
\hline $\begin{array}{c}\text { Diabetic } \\
\text { Maculopathy }\end{array}$ & Normal & Metamorphopsia & Total \\
\hline No maculopathy & 65 & 2 & 67 \\
\hline CSME & 3 & 0 & 3 \\
\hline Cystoid macular edema & 1 & 0 & 1 \\
\hline Total & 69 & 2 & 71 \\
\hline $\begin{array}{c}\text { P value on } \\
\text { chi-square test }\end{array}$ & & 0.940 & \\
\hline \multicolumn{4}{|c|}{ Comparison of Maculopathy with Amsler's Grid Evaluation } \\
\hline \multicolumn{4}{|c|}{ Table 6} \\
\hline
\end{tabular}

The Amsler's comparison with diabetic retinopathy showed that metamophopsia was seen in only two cases which were approximately around $2.81 \%$ of the study population. Chi-square test was applied for statistical analysis. The statistical analysis was non-significant with a $\mathrm{P}$ value of 0.298 . The Amsler's grid showed metamorphopsia in only 2 cases which actually did not have maculopathy. The association was non-significant with a P value of 0.940 .

\section{DISCUSSION}

It has been shown in literature that there is male-female differences in the occurrence of systemic diabetes. In a study by Glen et al. they found that in adolescence, the incidence of type 1 diabetes is greater in males, whereas in type 2 diabetes, the incidence is greater in females. However, it was seen that advanced retinopathy in type 1 diabetes appears to be more common in males and the presence and severity of diabetic retinopathy at the time of diagnosis in type 2 diabetes appears to be associated more with male sex. In our study, we had more male patients presenting with diabetic retinopathy as compared to females. Since our study is a hospital based study we had more male patients seeking help for diabetes compared to females. Probably the study by Glen Ozawa et al. was a population based study to calculate the incidence and prevalence of diabetes.

In a study by CH Tan et al. there were 60 cases (75.94\%) in the age group of 50 to 70 years. An Indian study had shown the mean age of presentation of diabetic retinopathy in their study as 56.69 years of age. In our study also the same age group was seen and we found that diabetic retinopathy was seen in 36 $(50.70 \%)$ cases. There was no evidence of diabetic retinopathy in 35 (49.29\%) cases. Amongst the retinopathy cases, the moderate type of diabetic retinopathy was highest amounting to $21(29.577 \%)$ cases. In the South Indian study by Nadarajan et al. the prevalence of diabetic retinopathy was found to be $32.53 \%$ for unilateral involvement and $31.58 \%$ for bilateral involvement. The incidence of moderate diabetic retinopathy was $51.9 \%$ in their study. Since ours was a hospital based study, we had higher cases of diabetic retinopathy compared to the study by Nadaraja et al.
Macular oedema in our case was seen only in $5.633 \%$ of cases. Amongst them we had $1.408 \%$ of cystoid macular oedema cases and $4.225 \%$ of clinically significant macular oedema. In our study, we used $90 \mathrm{D}$ biomicroscope to document the macular oedema. Cystoid macular oedema was diagnosed by evaluating the height of retinal blood vessels over the retinal pigment epithelium, loss of foveal depression and presence of cystoids spaces.

Clinically-significant macular edema (CSME) is defined as one or more of the following: retinal thickening at or within $500 \mu \mathrm{m}$ of the center of the macula; hard exudates at or within $500 \mu \mathrm{m}$ of the center of the macula, if associated with adjacent retinal thickening; or a zone or zones of retinal thickening one disc area in size, least part of which is within one disc diameter of the center of the macula. ${ }^{8}$

We had not used OCT in our study population. OCT is more often used for the detection and monitoring of DME. Literature has reported a weak to modest correlation between OCT measured center-point thickness and visual acuity. In Tables 6, 7 the photostress test is compared and presented in our study population. In our study abnormal PST (more than 50 seconds) was found only in 6 patients (8.45\%) though macular oedema was seen in our population in only $5.633 \%$ of cases. This proves that some of the non - macular oedema cases also showed a deranged macular functions. This will point to the fact that photostress test may be abnormal in sub clinical macular oedema probably detected only on OCT. Photostress recovery time increases with age but is proven to be independent of pupil size, ametropia and visual acuity.

Photostress recovery time (PSRT) describes the time required to regain normal visual function following exposure to intense light that bleaches the visual pigments and saturates the response of the macular photoreceptors and thereby causes a transient loss of vision.

The photo stress test predominantly helps in evaluation of pathologies affecting the retinal pigment epithelium photoreceptor complex. The lesions involving inner retinal layers may show a lesser abnormal recovery time compared to those involving the photoreceptor pigment epithelium complex. The magnitude of the PSRT reflects the efficiency with which the visual system recovers from exposure to a glare source and is principally dependent upon the integrity of the photoreceptors and the retinal pigment epithelium. PSRT deficits have been reported in asymptomatic subjects where visual acuity was good, indicating that a suitably designed test might provide an effective indicator of early disease or disease progression.

A study by Loughman et al. used a macular degeneration detector machine on their patients of diabetic retinopathy. This machine is based on photo stress test principle. They found in their study that PSRT measures using the MDD-2 device averaged in excess of 20 seconds, more than 3 times the average measures obtained for diabetic subjects without maculopathy. These allied findings suggest that the flash recovery device is sensitive to macular changes and furthermore, that photostress losses in patients with diabetes are particular to the development of diabetic maculopathy, the most common cause of visual impairment.

A study by Mallik et al. concluded that photostress test can be used for prognosis of visual recovery following macular involvement. They also found that the photo stress time returned to normalcy later compared to the other macular 
function tests. Also after this normalization, no further improvement in visual acuity was documented. A study by Magder et al. and Chilaris et al. concluded that if the retinal lesion shows a prolonged photostress test, then there exists some chances of visual recovery.

We found that there was a significant prolongation of PST with the duration of diabetes. The cases of maculopathy also showed a prolonged PST. This has been showed in table 4 . We also found that patients with moderate diabetic retinopathy had the maximum cases of abnormal PST, approximately 4 $(19.04 \%)$ patients having abnormal PST amongst 21 cases. In Table 7, 8, 9 we have compared the Amsler's grid test with diabetic retinopathy, duration of diabetes and diabetic maculopathy. None of the above said parameters were significantly associated with Amsler's grid changes.

A study by Michael et al. comparing the sensitivity and specificity of Amsler's grid with age related macular degeneration showed that the sensitivity of Amsler's charts for macular disease can be less than $50 \%$. The study explained this poor sensitivity with the phenomenon of perceptual completion whereby regular objects are "filled-in" across the scotoma. Another study has shown that sensitivity of Amsler's chart is as low as $56 \%$. Smaller scotomas less than 6 degrees remains undetected amounting to a false negative of $77 \%$.

Another study by Wolfe et al. suggested that the Amsler's grid being a suprathreshold testing methodology fails to detect a relative central scotoma. They suggested usage of low luminance threshold Amsler's grid testing which can detect scotomas in diabetic retinopathy. Our study did not find any significant change in Amsler's testing on patients with diabetic retinopathy, maculopathy or duration of diabetes.

\section{CONCLUSIONS}

We can conclude that photostress test can be used for detection of functional derangement of macula in cases of diabetes in pre and early clinical stages. The photo stress test prolonged recovery time was significantly associated with the time duration of presence of diabetes. The photo stress test was also found to be prolonged though within the normalcy range in cases of moderate diabetic retinopathy. The Amsler's grid test outcome was not found to be significantly associated with type of diabetic retinopathy, maculopathy or with the time duration of presence of diabetes.

\section{Limitation}

A larger sample size for significant association between photostress test and diabetic retinopathy and maculopathy will be needed. Low luminance Amsler's grid with tangent screen evaluation should be tried in diabetic retinopathy and maculopathy as a screening test.

Data sharing statement provided by the authors is available with the full text of this article at jemds.com.

Financial or other competing interests: None.

Disclosure forms provided by the authors are available with the full text of this article at jemds.com.

\section{REFERENCES}

[1] Lee R, Wong TY, Sabanayagam C. Epidemiology of diabetic retinopathy, diabetic macular edema and related vision loss. Eye Vis (Lond) 2015;2:17.

[2] Cheung N, Mitchell P, Wong TY. Diabetic retinopathy. Lancet 2010;376(9735):124-36.

[3] Bourne RRA, Stevens GA, White RA, et al. Causes of vision loss worldwide, 1990-2010: a systematic analysis. Lancet Glob Health 2013;1(6):e339-49.

[4] Yau JWY, Rogers SL, Kawasaki R, et al. Global prevalence and major risk factors of diabetic retinopathy. Diabetes Care 2012;35(3):556-64.

[5] Lightman S, Towler HM. Diabetic retinopathy. Clin Cornerstone 2003;5(2):12-21.

[6] Tong L, Vernon SA, Kiel W, et al. Association of macular involvement with proliferative retinopathy in type 2 diabetes. Diabet Med 2001;18(5):388-94.

[7] Musat O, Cernat C, Labib M, et al. Diabetic macularedema. Rom J Ophthalmol 2015;59(3):133-6.

[8] Photocoagulation for diabetic macular edema. Early Treatment Diabetic Retinopathy Study report number 1. Early Treatment Diabetic Retinopathy Study Research Group. Arch Ophthalmol 1985;103(12):1796-1806.

[9] Wu G, Weiter JJ, Santos S, et al. The macular photostress test in diabetic retinopathy and age-related macular degeneration. Arch Ophthalmol 1990;108(11):1556-8.

[10] Amsler M. Earliest symptoms of disease of the macula. $\mathrm{Br}$ J Ophthalmol 1953;37(9):521-37.

[11] Wall M, May DR. Threshold Amsler grid testing in maculopathies. Ophthalmology 1987;94(9):1126-33.

[12] Pandey AN, Raina A, Sharma PD. A study on Amsler's grid in acquired macular disorders. Ophthalmology Research: An International Journal 2016;6(3):1-7.

[13] Chalk D, Pitt M, Vaidya B, et al. Can the retinal screening interval be safely increased to 2 years for type 2 diabetic patients without retinopathy? Diabetes Care 2012;35(8):1663-8.

[14] Vijan S, Hofer TP, Hayward RA. Cost-utility analysis of screening intervals fordiabetic retinopathy in patients with type 2 diabetes mellitus. JAMA 2000;283(7):889-96.

[15] Klein R, Klein BE, Moss SE, et al. The wisconsin epidemiologic study of diabetic retinopathy. II. Prevalence and risk of diabetic retinopathy when age at diagnosis is less than 30 years. Arch Ophthalmol 1984;102(4):520-6.

[16] Sala SD, Bertoni G, Somazzi L, et al. Impaired contrast sensitivity in diabetic patients with and without retinopathy: a new technique for rapid assessment. $\mathrm{Br} \mathrm{J}$ Ophthalmol 1985;69(2):136-42.

[17] Sokol S, Moskowitz A, Skarf B, et al. Contrast sensitivity in diabetics with and without background retinopathy. Arch Ophthalmol 1985;103(1):51-4.

[18] Roy MS, Gunkel RD, Podgor MJ. Color vision defects in early diabetic retinopathy. Arch Ophthalmol 1986;104(2):225-8.

[19] Feitosa-Santana C, Oiwa NN, Paramei GV, et al. Color space distortions in patients with type 2 diabetes mellitus. Vis Neurosci 2006;23(3-4):663-8.

[20] Feitosa-Santana C, Paramei GV, Nishi M, et al. Color vision impairment in type 2 diabetes assessed by the D-15d test 
and the cambridge colour test. Ophthalmic Physiol Opt 2010;30(5):717-23.

[21] Palmowski AM, Sutter EE, Bearse MA Jr, et al. Mapping of retinal function in diabetic retinopathy using the multifocal electroretinogram. Invest Ophthalmol Vis Sci 1997;38(12):2586-96.
[22] Fortune B, Schneck ME, Adams AJ. Multifocal electroretinogram delays reveal local retinal dysfunction in early diabetic retinopathy. Invest Ophthalmol Vis Sci 1999;40(11):2638-51. 